Gut, 1980, 21, 192-194

\title{
Hydrolysis of pyridoxal-5'-phosphate in plasma in conditions with raised alkaline phosphatase
}

\author{
B B ANDERSON,* H O'BRIEN, G E GRIFFIN,* AND D L MOLLIN \\ From the Department of Haematolagy, St. Bartholomew's Hospital, London, and Department of Medicine, \\ Hammersmith Hospital, London
}

SUMMARY Hydrolysis of pyridoxal phosphate in plasma was demonstrated in patients with liver disease and other conditions with raised alkaline phosphatase, and this usually closely paralleled the alkaline phosphatase level, whether of liver or bone origin. The endogenous plasma pyridoxal phosphate was inversely related to the alkaline phosphatase, and plasma hydrolysis of pyridoxal phosphate may at least in part be responsible. Very large doses of vitamin $\mathbf{B}_{6}$ may be necessary to compensate for this hydrolysis.

Pyridoxal-5'-phosphate is the active coenzyme form of vitamin $\mathbf{B}_{6}$. It is also the main form in plasma circulating as an albumin bound complex. ${ }^{1-3}$ The plasma level of pyridoxal phosphate is commonly low in liver disease. ${ }^{4-6}$ The reason for this is uncertain but there is evidence that it may be due to an increased degradation of pyridoxal phosphate. ${ }^{56}$

Pyridoxal phosphate is known to be hydrolysed to pyridoxal by alkaline phosphatase. ${ }^{7}$ This also occurs when pyridoxal phosphate is bound to albumin, but at a slower rate. ${ }^{2}$ Dialysis studies have suggested that there is some free pyridoxal phosphate in equilibrium with the albumin-pyridoxal phosphate complex and this could explain why hydrolysis by alkaline phosphatase occurs in spite of binding to albumin. $^{2}$

We have investigated whether there is a significant hydrolysis of pyridoxal phosphate in the plasma of patients with a raised alkaline phosphatase.

\section{Methods}

Twenty-five subjects were studied. Ten were control subjects and were healthy members of the staff. Fifteen were patients with conditions associated with a raised alkaline phosphatase. Eleven of these had hepatobiliary disorders-namely, common bile duct obstruction (two), primary biliary cirrhosis (one), chronic active hepatitis (one), haemochromatosis (two), secondary carcinoma (one), hydatid cyst (one), drug-induced hepatitis (one), cirrhosis (one),

*Address for correspondence and reprints: Dr B B Anderson, Department of Haematology, St. Bartholomew's Hospital, West Smithfield, London EC1A 7BE.

Received for publication 16 October 1980 and peliosis hepatis (one). The other four had conditions associated with a raised bone alkaline phosphatase-namely, Paget's disease (two) and secondary carcinoma (two) (the primaries being carcinoma of prostate and breast).

Serum alkaline phosphatase was measured by the method of Kind and King as adapted to a Vicker's M300 (normal range 25-200 IU/l). Bone alkaline phosphatase was distinguished from liver alkaline phosphatase by electrophoresis and heat stability.

In order to measure plasma hydrolysis of pyridoxal phosphate, plasma from heparinised blood was incubated at $37^{\circ} \mathrm{C}$ for one hour with and without the addition of $148 \mathrm{ng} / \mathrm{ml}$ pyridoxal phosphate (equivalent to $100 \mathrm{ng}$ pyridoxal). The endogenous level of pyridoxal phosphate and pyridoxal in plasma, and the amount of pyridoxal formed from hydrolysis of pyridoxal phosphate incubated with plasma, was measured by Lactobacillus casei microbiological assay as we have described previously. ${ }^{8}{ }^{9}$ This assay is specific for pyridoxal. After acid hydrolysis pyridoxal phosphate is dephosphorylated to pyridoxal and can thus be assayed in this form. The endogenous pyridoxal phosphate level is the difference between the assays of untreated and acid hydrolysed plasma. The dephosphorylation of exogenous pyridoxal phosphate by plasma is measured as the increase in pyridoxal after in vitro incubation as described above.

\section{Results}

There was a rapid hydrolysis of pyridoxal phosphate to pyridoxal in plasma from patients with high alkaline phosphatase. A close correlation between 


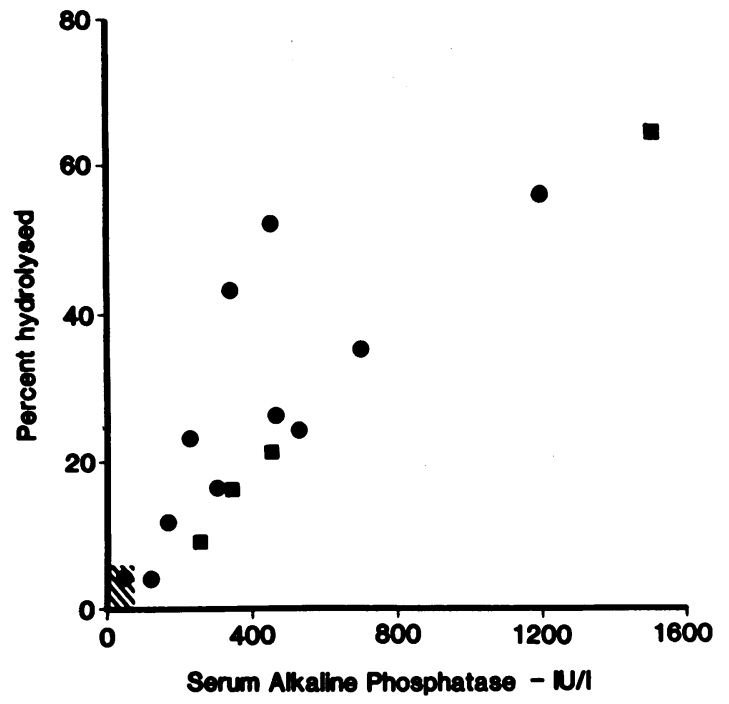

Fig. 1 The relationship between plasma hydrolysis of 148 ng pyridoxal phosphate per ml (equivalent to $100 \mathrm{ng}$ pyridoxal) to pyridoxal, and serum alkaline phosphatase level in 10 control subjects (hatched), 11 patients with a liver disorder (O), and four patients with a condition associated with increased bone alkaline phosphatase ( $\square$ ).

the amount of pyridoxal formed and the level of serum alkaline phosphatase is demonstrated in the 10 control subjects (represented by the hatched area) and the 15 patients detailed above (Fig. 1) $(r=0.9393$, $\mathbf{P}<0.001$ ). Serum alkaline phosphatase levels in healthy control subjects varied from 26-65 IU/1 and little or no pyridoxal phosphate was hydrolysed under these experimental conditions. In addition, there was little or no hydrolysis of pyridoxal phosphate in the plasma of two patients with liver disease but with normal serum alkaline phosphatase (haemochromatosis and cryptogenic cirrhosis). In the two patients with the highest phosphatase levels (one of liver origin and the other bone) $56 \%$ and $64 \%$ pyridoxal phosphate respectively was hydrolysed. The relationship of pyridoxal phosphate hydrolysis to the alkaline phosphatase level appeared to be the same whether the alkaline phosphatase was of liver or bone origin (Fig. 1). However, in the two patients with common bile duct obstruction the hydrolysis appeared relatively greater for the level of alkaline phosphatase.

In view of the binding of pyridoxal phosphate to albumin in plasma, the albumin was measured. The amount of hydrolysis at one hour did not bear any relationship to these levels, which ranged from $26 \mathrm{~g} / 1$ to $47 \mathrm{~g} / \mathrm{l}$.

There is a close inverse relationship between the

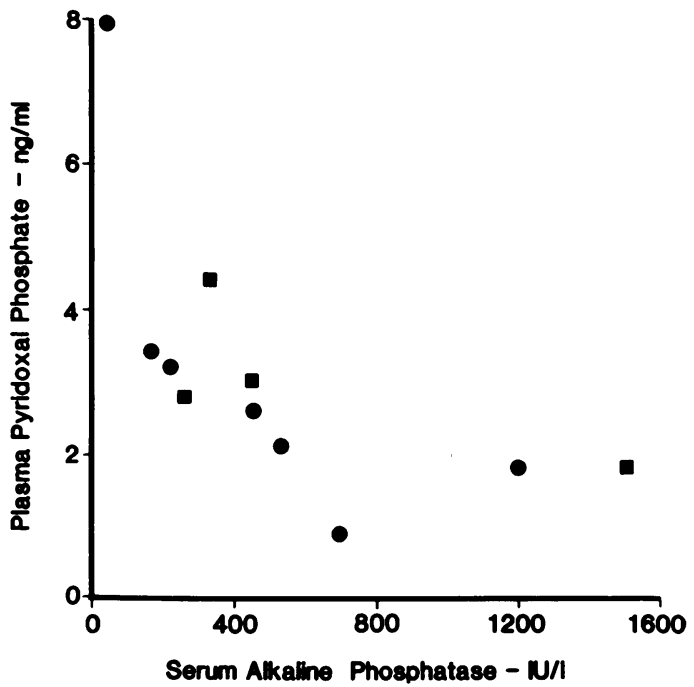

Fig. 2 The relationship between endogenous plasma pyridoxal phosphate (in terms of pyridoxal) and serum alkaline phosphatase level in seven patients with a liver disorder (O) and four patients with a condition associated with increased bone alkaline phosphatase

endogenous plasma pyridoxal phosphate and the alkaline phosphatase level in 11 patients (Fig. 2) $(r=-0.893, \mathrm{P}<0.001)$. The other four patients had been taking mixed vitamins containing pyridoxine and consequently had higher $B_{6}$ levels. The patient with the lowest pyridoxal phosphate level (Fig. 2) had neurological and haematological signs of $\mathbf{B}_{6}$ deficiency (Griffin et al., in preparation).

\section{Discussion}

The evidence that low plasma levels of pyridoxal phosphate might be explained by increased degradation of pyridoxal phosphate in liver disease was based on observations in normal subjects and patients with liver disease after intravenous pyridoxal phosphate. There was a smaller rise in plasma pyridoxal phosphate and a greater urinary excretion of vitamin $\mathbf{B}_{6}$ derivatives in patients with liver disease. ${ }^{5}{ }^{6}$ Mitchell et al. ${ }^{5}$ proposed that $\mathrm{B}_{6}$ degradation took place in the liver because they were unable to demonstrate increased degradation of pyridoxal phosphate in vitro in plasma of patients with liver disease. However, they appear to have studied only two patients and one control subject.

We have demonstrated in vitro in the plasma of patients with a raised alkaline phosphatase that pyridoxal phosphate is hydrolysed to pyridoxal, and that the amount of pyridoxal formed closely parallels the alkaline phosphatase level (Fig. 1). This might, 
in part, explain the low endogenous levels of pyridoxal phosphate reported in liver disease; in fact we have shown that there is a close inverse relationship between the plasma levels and the alkaline phosphatase (Fig. 2). The physiological significance of these findings is, however, uncertain; nor is it known how much hydrolysis of pyridoxal phosphate occurs in the liver of normal subjects or patients with liver disease.

It is possible that some of the pyridoxal formed from plasma hydrolysis of pyridoxal phosphate could be re-utilised, for it might be taken up by the tissues and subsequently rephosphorylated. ${ }^{2}{ }^{910}$ It is not certain, therefore, whether hydrolysis of pyridoxal phosphate in plasma would necessarily cause severe depletion in the body. However, one subject with long-standing liver disease and a grossly raised level of alkaline phosphatase presented clinically with epileptiform attacks and sideroblastic anaemia, both of which responded to large doses of pyridoxine (Griffin et al., in preparation). In addition, the observations of Ellis and Presley ${ }^{11}$ suggest that a subclinical deficiency of $\mathrm{B}_{6}$ may not commonly be recognised. It seems, therefore, reasonable that vitamin $\mathbf{B}_{6}$ should be included in the nutritional management of patients with liver disease as suggested by Labadarios et al. ${ }^{6}$ In fact, patients with a grossly raised level of alkaline phosphatase might require large doses of vitamin $B_{6}$ to compensate for hydrolysis.

The work was supported by a grant from the Wellcome Trust. We are grateful to Dr A M Dawson for allowing us to study some of his patients and thank Anne Frawley for her help with the assays.

\section{References}

${ }^{1}$ Dempsey WB, Christensen HN. The specific binding of pyridoxal-5'-phosphate to bovine plasma albumin. J Biol Chem 1962; 237: 1113-20.

${ }^{2}$ Lumeng L, Brashear RE, Li TK. Pyridoxal-5'-phosphate in plasma: source, protein-binding, and cellular transport. J Lab Clin Med 1974; 84: 334-43.

${ }^{3}$ Anderson BB, Nemark PA, Rawlins M, Green R. Plasma binding of vitamin $\mathrm{B}_{6}$ compounds. Nature 1974; 250: 502-4.

${ }^{4}$ Leevy CM, Thompson A, Baker H. Vitamins and liver injury. Am J Clin Nutr 1970; 23: 493-8.

${ }^{5}$ Mitchell DJ, Wagner C, Stone WJ, Wilkinson GR, Schenker S. Abnormal regulation of plasma pyridoxal5 '-phosphate in patients with liver disease. Gastroenterology 1976; 71 : 1043-49.

${ }^{6}$ Labadarios D, Rossouw JE, McConnell JB, Davis M, Williams $R$. Vitamin $\mathbf{B}_{6}$ deficiency in chronic liver disease-evidence for increased degradation of pyridoxal-5'-phosphate. Gut 1977; 18: 23-7.

${ }^{7}$ Turner JM. Pyridoxal phosphate breakdown by an alkaline-phosphatase preparation. Biochem $J 1961$ 80: 663-8.

${ }^{8}$ Anderson BB, Peart MB, Fulford-Jones CE. The measurement of serum pyridoxal by a microbiological assay using Lactobacillus casei. J Clin Pathol 1970; 23: $232-42$.

${ }^{9}$ Anderson BB, Fulford-Jones CE, Child JA, Beard MEJ, Bateman CJT. Conversion of vitamin $\mathbf{B}_{6}$ compounds to active forms in the red blood cell. J Clin Invest 1971; 50: 1901-9.

${ }^{10}$ Anderson BB. Red-cell metabolism of vitamin $B_{6}$. In: Tryfiates GP, ed. Vitamin $B_{6}$. Its metabolism and influence on the processes of growth. Food Science and Nutrition, 1979.

${ }^{11}$ Ellis JM, Presley J. Vitamin $\mathrm{B}_{6}$. In: The Doctor's Report. Harper and Row, New York 1973. 